\title{
Estimated use of abacavir among adults and children enrolled in public sector antiretroviral therapy programmes in Gauteng Province, South Africa
}

\author{
Denise Evans, Mhairi Maskew, Cassidy Heneger, Ian Sanne
}

Health Economics and Epidemiology Research Office, Department of Internal Medicine, Faculty of Health Sciences, University of the Witwatersrand, Johannesburg

Denise Evans, $\mathrm{PhD}$

Mhairi Maskew, MB BCh, MSc

Department of Epidemiology, Gillings School of Global Public Health, University of North Carolina at Chapel Hill, USA

Cassidy Heneger, $\mathrm{PhD}$

Clinical HIV Research Unit, Department of Internal Medicine, Faculty of Health Sciences, University of the Witwatersrand, and Right to Care, Johannesburg

Ian Sanne, MB BCh, FCP (SA), FRCP (Lond)

Corresponding author: D Evans (devans@witshealth.co.za)

In South Africa, abacavir $(\mathrm{ABC})$ is currently recommended as part of first- and second-line antiretroviral therapy (ART) for HIV-positive paediatric patients. Concerns about overprescribing of the drug, particularly to adults, led to an analysis of $\mathrm{ABC}$ use in public sector ART programmes. We investigated current prescription of the drug to adults and children accessing ART in 4 public sector programmes across Gauteng Province, South Africa. ABC was almost exclusively prescribed to children initiating ART and adults requiring regimen changes due to drug toxicities. Patterns of ABC use among HIV-positive paediatric patients followed national ART treatment guidelines on the application of the drug. Although $\mathrm{ABC}$ is commonly used in the private sector for adults, the current national ART treatment guidelines for adults and adolescents should include $\mathrm{ABC}$ as an alternative to standard first- or second-line ART.

S Afr J HIV Med 2012;13(3):134-137. DOI:10.719/SAJHIVMED.822

Abacavir (ABC), a nucleoside reverse transcriptase inhibitor (NRTI), has been shown to be an effective component of combination antiretroviral therapy (ART) regimens for both paediatric and adult patients. ${ }^{1-3}$ South African (SA) national ART treatment guidelines recommend $\mathrm{ABC}$ for first- and second-line regimens in paediatric patients aged $\geq 3$ months. ${ }^{4}$ Despite demonstrating a durable antiretroviral response, $\mathrm{ABC}$ is not a preferred option for first- or second-line adult regimens in SA, primarily due to its relatively high cost, but also due to concerns about toxicity, particularly hypersensitive reactions, although these are only experienced in $4 \%$ of individuals receiving ABC. ${ }^{5-7}$ Such reactions commonly present as a rash and fever, but may manifest as fatigue, flu-like symptoms or gastrointestinal upset. Common adverse effects include headache, loss of appetite, nausea and diarrhoea. ${ }^{7}$ Of all nucleoside reverse transcriptase inhibitors (NRTIs), $A B C$ is associated with the lowest rate of mitochondrial dysfunction, including lactic acidosis, peripheral neuropathy and lipoatrophy. ${ }^{5}$ Substitution of stavudine ( $\left.\mathrm{d} 4 \mathrm{~T}\right)$ with $\mathrm{ABC}$ improves mitochondrial indices, reduces adipocyte apoptosis, ${ }^{8}$ and has been shown to be superior to substitution with zidovudine (ZDV) in adults. In older children, once-daily use of $\mathrm{ABC}$ has also been shown to be effective, facilitating adherence and improving patient satisfaction. ${ }^{9-11} \mathrm{ABC}$-containing regimens are used in adult cases where standard first- and second-line treatments have either failed or cannot be tolerated.

Given current national guidelines for limited inclusion of ABC in highly active ART (HAART) regimens, use of the drug among HIV-positive adults eligible for treatment is generally expected to be low. In 2011, however, a higher than anticipated use of $\mathrm{ABC}$ was observed across all provinces of SA. Actual use outpaced the projected need for the drug, placing strains on supplies and programme budgets. In response to concerns about the unnecessary use of $\mathrm{ABC}(300 \mathrm{mg})$, we investigated current prescribing of the drug to adults and children accessing ART in public sector programmes across Gauteng Province.

\section{Methods}

In 2011 we performed a cross-sectional study of 4 urban public sector antiretroviral clinics in Gauteng Province supported by Right to Care - a non-governmental organisation supporting ART rollout in SA with funding from the United States Agency for International Development (USAID)/ 
President's Emergency Plan for AIDS Relief (PEPFAR). ${ }^{12}$ The clinics operate according to SA national ART guidelines and are run by the national Department of Health, as part of its development of accredited Comprehensive Care, Management and Treatment (CCMT) sites with doctor-managed initiation and follow-up. ${ }^{13,14}$ Age categories were defined according to the World Health Organization (WHO) for paediatrics ( $<10$ years of age), adolescents (aged $10-18$ years) and adults (aged $>18$ years). ${ }^{15}$ Medical records were reviewed for all patients actively receiving HAART at the Themba Lethu Clinic (TLC) in central Johannesburg (JHB) and at 3 other adjacent sites to the north, east and west of TLC. Data on current drug regimens, patient demographics and clinical characteristics were extracted from TherapyEdge-HIV ${ }^{\mathrm{TM}}$ (TE), an electronic patient management system used at the clinics. Use of TE data was approved by the Human Research Ethics Committee of the University of the Witwatersrand (HRECMedical M060626/M110140).

\section{Results}

Of 23084 patients, 76\% (22 089) were adults and 5\% (995) were children and adolescents (aged $\leq 18$ years), reflecting that the HIV clinics were mainly adult-orientated. The majority of patients attending the central-JHB (12 120/12 185; 99.5\%), west-JHB (1 875/2 267; 82.7\%) and east-JHB (2 705/2 773; 97.5\%) clinics were adults aged $>18$ years. TLC had a small cohort of paediatric ( $<10$ years) and adolescent (10 18 years) patients (65/12 185; 0.5\%), compared with north-JHB, where paediatric/adolescent (470/5 859; 8.0\%) and adult HIV-positive patients $(5389 / 5859 ; 92 \%)$ were seen separately (Table 1).

The total number of patients receiving ABC was 619/23 084 (2.7\%). An estimated 0.9\% (206/22 089) of adults attending the 4 sites were prescribed $\mathrm{ABC}$-containing regimens at the time of the study, compared with $42 \%$ (413/995) of paediatric/adolescent patients $(\leq 18$ years). Among patients aged $\leq 18$ years, $11 \%$ (7/65), 54\% (254/470), 33\% (128/392) and $35 \%(24 / 68)$ were receiving ABCcontaining regimens at the central-, north-, west- and east-JHB clinics, respectively. At the west- and east-JHB clinics, nearly all patients receiving $\mathrm{ABC}(98 \% ; 128 / 131$ and $96 \%$; $24 / 25$, respectively) were aged $\leq 18$ years. The number of adults receiving $\mathrm{ABC}$ varied from $2.3 \%(3 / 131)$ in west-JHB, to $4 \%(1 / 25)$ in east-JHB, 27\% (95/349) in north-JHB and 94\% $(107 / 114)$ in central-JHB. Despite the small size of some cohorts, the results demonstrated a variation in the number of adults prescribed $\mathrm{ABC}$ at these 4 clinics.

Among paediatric and adolescent patients (aged $\leq 18$ years), median time receiving ART and $A B C$ varied by clinic. The TLC paediatric/ adolescent population had a median duration on ART of 91 months (IQR 40 - 94 months) compared with 37 months (IQR 24 - 51) at the north-JHB clinic and 6 months at both the west-JHB (IQR 3 - 27 months) and eastJHB (IQR 2 - 8 months) clinics. A longer median duration of ART was associated with longer time receiving $\mathrm{ABC}$, with paediatric/ adolescent patients at TLC prescribed the drug for a median 40 months (IQR 20 - 94 months). The median time on $\mathrm{ABC}$ was shorter at the north-JHB (13 months; IQR 6 - 24 months), west-JHB (5 months; IQR 2 - 8 months) and east-JHB clinics (5 months; IQR 2 - 6 months). More than half of the paediatric/adolescent patients at TLC $(4 / 7 ; 57 \%)$ and the northJHB clinic (129/254; 51\%) had been receiving $\mathrm{ABC}$ for longer than 12 months. Among adult patients receiving $A B C$ at the clinics at the time of the study, $35 \%$ (TLC; 37/107) and 73\% (north-JHB; 69/95) had been receiving the drug for longer than 1 year. Only 6\% (8/128) of patients attending the west-JHB clinic were prescribed $\mathrm{ABC}$ for longer than 12 months.

The majority of adult patients prescribed $\mathrm{ABC}$ at the time of the study were switched onto the drug from other regimens, with only $18 \%(19 / 107)$ of adult patients at TLC and $28 \%(27 / 95)$ of adult patients at north-JHB clinic initiated on an ART regimen containing $\mathrm{ABC}$. Among adult patients reporting a reason for switching to $\mathrm{ABC}$ from another regimen (52\%; 81/156), the most common reasons were peripheral neuropathy $(17 \%, 14 / 81)$, abnormal fat distribution $(28 \%, 23 / 81)$ and toxicity $(35 \%$, 28/81). Few specific reasons for switching to an $\mathrm{ABC}$-containing regimen were given for paediatric/adolescent patients $(25 \%, 62 / 247)$, but among those cases where a reason was reported, abnormal fat distribution was the leading cause of regimen change $(76 \%, 47 / 62)$

\section{Discussion}

When considering sites where both adults and children are routinely treated with $\mathrm{ART}, \mathrm{ABC}$ is primarily prescribed to children. At the eastand west-JHB clinics, the drug was almost exclusively provided to patients aged $\leq 18$ years. We demonstrated a high variation in the prescription of $\mathrm{ABC}$ to adult and paediatric/ adolescent patients at the 4 sites across Gauteng Province. While we did not demonstrate dramatic over-use of the drug, $67 \%$ of patients receiving $\mathrm{ABC}$ were children, consistent with guidelines, while $33 \%$ were adults, as a likely result of being switched to $A B C$ from another ART regimen due to adverse effects or toxicity.

However, approximately 23\% (46/202) of adults at central- and north-JHB were initiated on an $\mathrm{ABC}$-containing regimen. $\mathrm{A}$ large proportion of $\mathrm{ABC}$ users at the northJHB clinic (73\%; 254/349) were paediatric/ adolescent patients compared with a smaller proportion $(27 \%$; $95 / 349)$ of adults, yet still less than $2 \%(95 / 5859)$ of patients aged $\geq 18$ years were on an $\mathrm{ABC}$-containing regimen. A large proportion of these adult patients receiving $\mathrm{ABC}$ reported long-term use of the drug (73\% for 1 year or longer). The large number of adult patients receiving $\mathrm{ABC}$ at the northJHB clinic was likely related to being switched onto $\mathrm{ABC}$ from another $\mathrm{ART}$ regimen due to an adverse effect (i.e. peripheral neuropathy, lactic acidosis or lipoatrophy/lipodystrophy) less than $30 \%$ of patients aged $\geq 18$ years were initiated on an $\mathrm{ABC}$-containing regimen.

The majority of paediatric/adolescent patients $(<80 \%)$ at the north-JHB clinic were switched onto an $\mathrm{ABC}$-containing regimen, with 19\% (39/201) being due to abnormal fat redistribution. Once-daily use of $\mathrm{ABC}$ has been shown to be effective in older children, thereby facilitating adherence and improving patient satisfaction; and it is therefore possible that specialist paediatricians at north-JHB might have prescribed/switched adolescents to $\mathrm{ABC}$ for adherence issues. ${ }^{10,11}$ Twenty-one per cent of paediatric/adolescent patients were initiated on an $\mathrm{ABC}$-containing regimen. $\mathrm{A}$ large proportion of these patients reported long-term use of the drug, with $51 \%$ (129/254) receiving $\mathrm{ABC}$ for longer than 1 year. This is likely due to the ageing paediatric population receiving care which has maintained paediatric regimens even after moving into adolescence (age 10 - 18 years).

Conversely, <1\% (107/12 120) of adults at TLC were receiving $A B C$, only $35 \%$ (37/107) of whom had received the drug for longer than 1 year. Adults were unlikely $(17.8 \%$; 19/107) to be initiated on an $\mathrm{ABC}$-containing regimen but rather switched to such a regimen due to toxicity $(82.2 \%$; 88/107). The CD4 count (267 cells $/ \mathrm{mm}^{3}$; IQR 160 - 392) and proportion with a detectable viral load $(13 \%$; 10/80) at the initiation of $\mathrm{ABC}$ confirmed that adults patients were most likely switched to $A B C$ rather than initiated on the drug. TLC treats few patients aged $<18$ years $(0.5 \%$ of total patients); however, $71 \%(5 / 7)$ of paediatric/ 


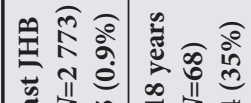

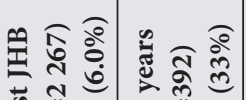

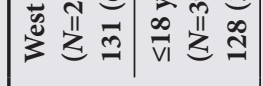

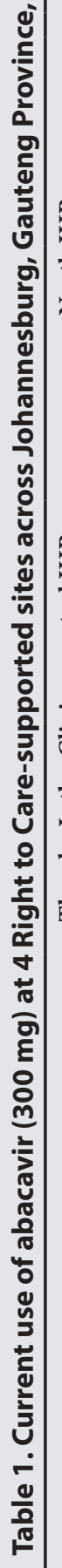

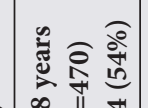

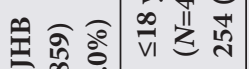
i

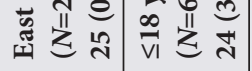

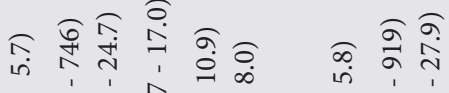

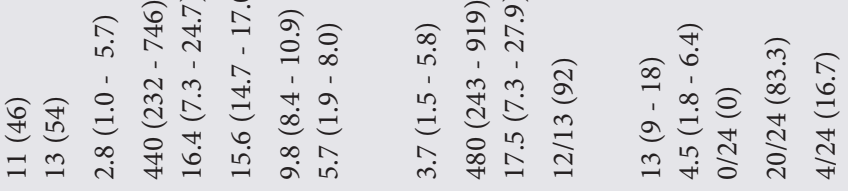

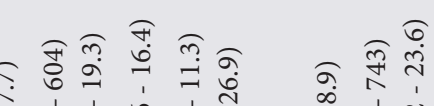

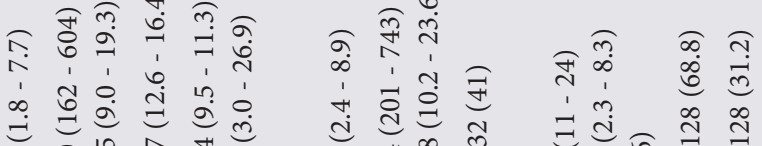

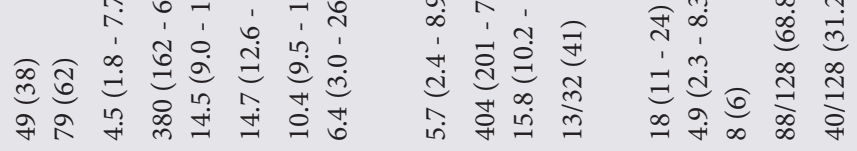
กิ

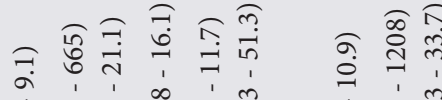

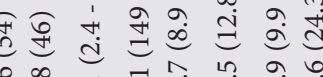

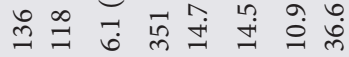

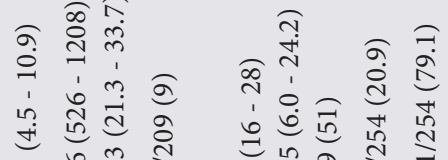

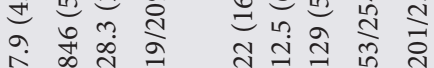

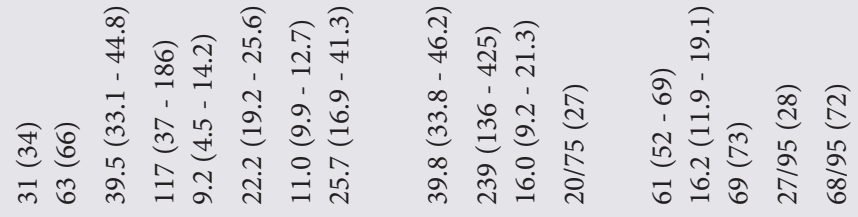

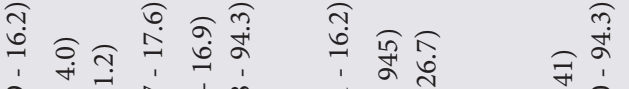

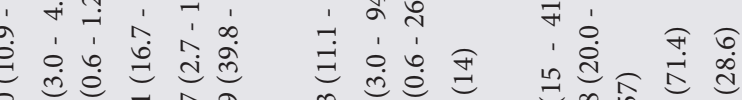

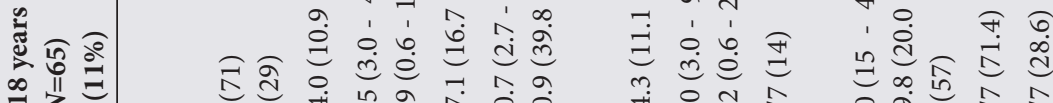

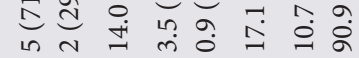

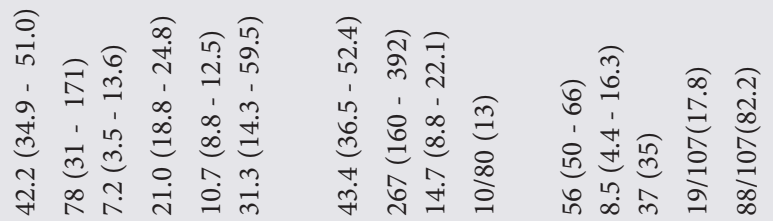

\section{急}

๙ิ

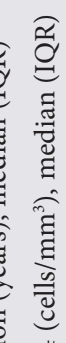

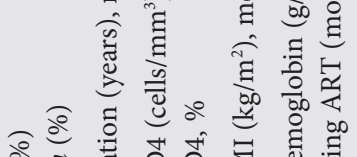

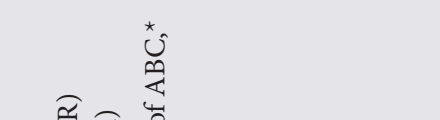

参䄈

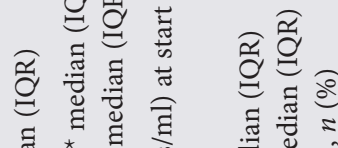

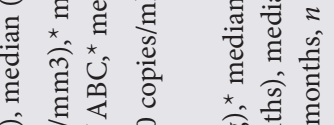

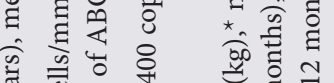

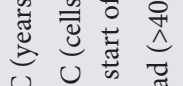

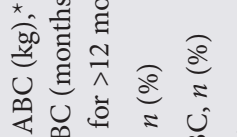

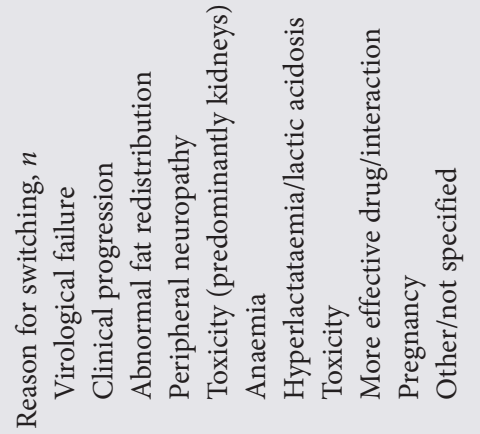


adolescent patients on an $\mathrm{ABC}$-containing regimen were initiated on such a regimen.

Differences in $\mathrm{ABC}$ use across clinics possibly also partially reflect differences in clinical practice. More than $50 \%$ of children from the north-JHB clinic were receiving $\mathrm{ABC}$ for longer than 1 year, compared with $16 \%$ and $0 \%$ at west- and east-JHB, respectively. This could be a reflection of advice and recommendations concerning treating ART side-effects, from specialist paediatric HIV physician services available at north-JHB clinic (not available at the other sites). This advice might have included switching from stavudine- to $\mathrm{ABC}$-containing regimens before implementation of the new national ART treatment guidelines. The switching of many children to $\mathrm{ABC}$ at north-JHB (79.1\%; 201/254) before implementation of the national guideline could explain the perception of high consumption of this drug in JHB.

The majority (79\%) of children from the north-JHB clinic were switched onto $\mathrm{ABC}$ from another regimen, while $69 \%$ and $83 \%$ of children from west- and east-JHB, respectively, were initiated onto $\mathrm{ABC}$. This likely reflects the implementation of the 2010 SA national ART treatment guidelines at these 2 sites, which recommend that infants and children be initiated on a regimen of $A B C$ and lamivudine (3TC) plus lopinavir/ritonavir (LPV/r) or efavirenz (EFV). ${ }^{16}$ Among paediatric and adolescent patients, the most frequent reason for switching onto an $\mathrm{ABC}$-containing regimen was ART toxicity and side-effects, most commonly abnormal fat redistribution. This highlights the toxicity of NRTIs, particularly $\mathrm{d} 4 \mathrm{~T}$, didanosine (ddI) and zidovudine (AZT), even in children. ${ }^{7}$

The majority of adults prescribed $\mathrm{ABC}$ at the time of the study had been switched onto the drug from other regimens. Specified reasons for prescribing $A B C$ to these adults were largely related to ART toxicity and side-effects, including renal toxicity, anaemia, peripheral neuropathy, abnormal fat distribution, and hyperlactataemia or lactic acidosis. This highlights the need to make $\mathrm{ABC}$ available to adults and include the drug in the national guidelines for adults as an alternative to standard first- and second-line ART.

\section{Study strengths and limitations}

The biggest strengths of this study include the size of the cohort and the depth of the data (using a standardised, electronic datacapturing system, data on ART regimens, visit dates, outcomes, laboratory investigations and demographic data is of high quality)..$^{12}$ Since these are clinical cohorts, the findings should be considered in light of the study limitations which include missing data (laboratory investigations and reasons for switching) or under-reported conditions or adverse drug effects and the generalisability of the results. This study was done in RTC-supported sites in Johannesburg, thus with a higher level of clinical support and progressive prescription than other sites (i.e. rural sites without any supporting NGO). It is likely that $\mathrm{ABC}$ use, especially in adults, would be lower in rural, unsupported sites.

\section{Conclusion}

After reviewing the data, there did not appear to be a dramatic over-use of $\mathrm{ABC}(300 \mathrm{mg})$ in the 4 Gauteng sites. At the time of the study, there were 619 patients on ABC-containing regimens, accounting for only $2.7 \%$ of all the patients currently receiving ART at these clinics. Further, patterns of use at the 4 sites appeared in line with national ART treatment guidelines; most patients prescribed $\mathrm{ABC}$ were paediatric patients or adults experiencing drug toxicities on non- $\mathrm{ABC}$-containing regimens.

Conflict of interest. The authors declare that no competing interests exist. Right to Care (RTC) funded part of the research and supported the provision of treatment for the study's patients.

Acknowledgments. We acknowledge the directors and staff of Themba Lethu Clinic (TLC), CHRU and Right to Care (RTC) - a PEPFAR-funded NGO. We wish to acknowledge the Gauteng and national Departments of Health for providing for the care of the patients as part of the National Comprehensive Care, Management and Treatment (CCMT) of HIV and AIDS programme. Sincere thanks to the patients attending the RTC-supported clinics for their continued trust in the treatment and care provided at the clinic.

Funding. Funding was provided by United States Agency for International Development (USAID) under the terms of agreement 674A-00-08-00007-00 with Right to Care (RTC). $\mathrm{D}$ Evans is supported by the Claude Leon Foundation and NIH/CFAR/IAS Creative and Novel Ideas in HIV Research (CNIHR) programme (sub-award with UAB Center for AIDS Research: P30AI027767). The opinions expressed herein are those of the authors and do not necessarily reflect the views of the NIH,
NIAID, USAID, the Themba Lethu Clinic or Right to Care. Right to Care provided funding for technical and logistical support and for the provision of treatment for patients in this study.

\section{References}

1. Henry K, Wallace RJ, Bellman PC, et al. Twicedaily triple nucleoside intensification treatment with lamivudine-zidovudine plus abacavir sustains suppression of human immunodeficiency virus type 1: Results of the TARGET study. J Infect Dis 2001;183:571-578

2. Green H, Gibb DM, Walker AS, et al. Lamivudine/ abacavir maintains virological superiority over zidovudine/lamivudine and zidovudine/abacavir beyond 5 years in children. AIDS 2007;21:947-955.

3. Saez-Llorens X, Nelson RPJ, Emmanuel P, et al. A randomized, double-blind study of triple nucleoside therapy of abacavir, lamivudine, and zidovudine versus lamivudine and zidovudine in previously treated human immunodeficiency virus type 1-infected children. Paediatrics 2001;107:E4. [http:// dx.doi.org/10.1542/peds.107.1.e4]

4. Serenata C. Changes to the ART guidelines - an overview. Southern African Journal of HIV Medicine 2010;11:28-30

5. Rabie H, Henning KL, Schoeman P, de Villiers N, Pretorius GHJ, Cotton MF. Abacavir: its use and hypersensitivity. Southern African Journal of HIV Medicine 2009;10(4):81-84.

6. Hewitt R G. Abacavir hypersensitivity reaction. Clin Infect Dis 2002;34(8):1137-1142. [http://dx.doi. org/10.1086/339751]

7. Orrell C. Antiretroviral adverse drug reactions and their management. Continuing Medical Education 2011;29:234-237.

8. McComsey GA, Paulsen DM, Lonergan JT, et al. Improvements in lipoatrophy, mitochondrial DNA levels and fat apoptosis after replacing stavudine with abacavir or zidovudine. AIDS 2005;19:15-23.

9. Carr A, Workman C, Smith DE, et al. Abacavir substitution for nucleoside analogs in patients with HIV lipoatrophy: a randomized trial. JAMA 2002;288:207-215. [http://dx.doi.org/10.1001/2012. jama.10158]

10. Scherpbier HJ, Bekker V, Pajkrt D, Jurriaans S, Lange JM, Kuijpers TW. Once-daily highly active antiretroviral therapy for HIV-infected children: safety and efficacy of an efavirenzcontaining regimen. Pediatrics 2007;119:e705-715. [http://dx.doi.org/10.1542/ peds.2006-1367]

11. LePrevost M, Green H, Flynn J, et al. Adherence and acceptability of once daily lamivudine and abacavir in human immunodeficiency virus type-1 infected children. Pediatr Infect Dis J 2006;25:533-537.

12. Fox MP, Maskew M, MacPhail P, et al. Cohort profile: The Themba Lethu Clinical HIV Cohort, Johannesburg, South Africa, 2012. International Journal of Epidemiology. In press. [http://dx.doi. org/10.1093/ije/dys029]

13. National Department of Health (DoH). The South African Antiretroviral Treatment Guidelines. Pretoria: DoH, 2010.

14. World Health Organization. Antiretroviral therapy for HIV infection in adults and adolescents, recommendations for a public health approach: 2010 revision. Geneva: WHO, 2010. http://whqlibdoc. who.int/publications/2010/9789241599764_eng.pdf (accessed 1 February 2012)

15. World Health Organization (WHO). Towards Universal Access: Scaling up priority HIV/AIDS interventions in the Health Sector. Progress Report April 2007. Geneva, Switzerland: WHO Press, 2007.

16. Department of Health, South Africa. 2010. Guidelines for the management of HIV in children, 2nd Edition 2010. http://www.hivfshealth.org/sites/default/files/ ART\%20guidelines\%20paeds\%202010.pdf (accessed 5 June 2012) 